\title{
El Licenciado en Contaduría y su participación en el desarrollo
} de las Mipymes. The Bachelor of Accounting and participation in the development of Mipymes

Roger Manuel Patrón Cortés. Universidad Autónoma de Campeche roger_patron_cortes@hotmail.com

Luis Alfredo Argüelles Ma. Universidad Autónoma de Campeche luisarguellesmaa@hotmail.com

\section{Carlos Alberto Pérez Canul.} Universidad Autónoma de Campeche cperezxx@msn.com

\section{Resumen}

Esta investigación tuvo como objetivo determinar si los Licenciados en Contaduría de la Facultad de Contaduría y Administración de la Universidad Autónoma de Campeche prefieren crear empresas o trabajar por cuenta ajena dentro del sector público o privado. Este trabajo fue exploratorio de corte cualitativo. El 31 \% de los Licenciados en Contaduría trabajan en el gobierno y el $21 \%$ en la asesoría contable y financiera. Se encontró que el 14 \% de los encuestados tiene una empresa propia y el 86 \% trabaja por cuenta ajena. Lo que indica que se tiene un nivel muy bajo de la actividad emprendedora y por tanto del desarrollo económico empresarial del estado de Campeche. Los primeros manifestaron como fortaleza sus 
conocimientos administrativos y la elaboración de proyectos mientras que los segundos sus conocimientos contables y fiscales. El primer grupo manifestó como debilidades la falta de conocimientos de software de cómputo y la falta de actualización, mientras que el segundo la falta de actualización en diversas materias que cursaron. Ambos grupos manifestaron que el principal problema para iniciar una empresa es la falta de capital, sin embargo, también opinaron que no todos los profesionistas tienen que ser emprendedores para ser socialmente responsables y contribuir al desarrollo económico del estado de Campeche y de México.

Palabras clave: Licenciado en Contaduría, desarrollo económico, Mipymes.

\section{Abstract}

This study aimed to determine whether graduates of the School of Accounting and Management Accounting Autonomous University of Campeche prefer to create businesses or work as an employee in the public or private sector. This work was exploratory qualitative. $31 \%$ of graduates in Accounting work in government and $21 \%$ in accounting and financial advice. It was found that $14 \%$ of respondents have a company and $86 \%$ work for others. This indicates that there is a very low level of entrepreneurial activity and economic development in both business Campeche state. The first showed its strength as administrative expertise and project development while the latter its accounting and tax knowledge.

Key words: Degree in Accounting, economic development, MiPYMES

Fecha recepción: Marzo 2013 Fecha aceptación: Junio 2013

\section{Introducción}

Este trabajo tiene como objetivo investigar a los Licenciados en Contaduría (LC) de la Facultad de Contaduría y Administración (FCA) de la Universidad Autónoma de Campeche (UAC), con relación a sus decisiones laborales para crear sus propias fuentes de trabajo, o bien, para trabajar por cuenta ajena en una empresa privada o en la administración pública; así como 
también, encontrar los principales motivos de su decisión. Además, tiene como objetivos específicos: a) Identificar los motivos de su decisión para trabajar en una empresa, b) Identificar las fortalezas y debilidades de su preparación, y c) Determinar los principales problemas que enfrentan y cómo los manejan y resuelven. El trabajo presenta primero los antecedentes, posteriormente explica el método de investigación para la recolección de datos y el análisis de los resultados, finalmente expone conclusiones y recomendaciones del mismo.

\section{1. Antecedentes.}

La Universidad del Sudeste, actualmente Universidad Autónoma de Campeche estableció el 26 de agosto de 1965 la Escuela Superior de Comercio de acuerdo al Artículo 4 de su Ley Orgánica. Se tomaron como base los planes y programas de estudio 1964 de la Universidad Nacional Autónoma de México (UNAM). En 1985 adoptó el Plan de Estudios Nacional, derivado de los trabajos realizados por la UNAM y la Asociación Nacional de Facultades y Escuelas de Contaduría y Administración (ANFECA), pasando de la modalidad anual a la semestral. En 1988 se convirtió en Facultad con la creación de la División de Posgrado y la Maestría en Contaduría. En 1990 se implementaron las especialidades en Fiscal e Informática, en 1993 el Diplomado en Proyectos de Inversión y la Especialidad en Comercio Exterior y en 1994 la Especialidad en Finanzas. El plan de estudios 1994 se fundamentó en el Plan Nacional 1993 producto de las universidades e instituciones de educación superior que integran la ANFECA. En 1994 y 1997 se adoptaron nuevos planes adicionando las materias de Redacción I y II, también se reubicaron las asignaturas y los créditos. De acuerdo con diversos estudios socio económicos realizados en la región y con el apoyo de académicos de la Facultad, empresarios y dependencias gubernamentales se realizaron los cambios siguientes: a) actualización del Plan de Estudios de la Carrera de Contador Público versión 2000, b) apertura de la Licenciatura en Administración y Finanzas, c) apertura de la Maestría en Administración con tres terminales, d) la carrera de Contador Público cambia a la de Licenciatura en Contaduría, y e) planes flexibles 2006 (Universidad Autónoma de Campeche, 2011).

Actualmente para la Licenciatura en Contaduría opera el plan de estudios 2009. Este plan tiene las 
características de ser flexible, por competencias, centrado en el aprendizaje y además se encuentra estructurado en 8 semestres con un mínimo de 51 unidades de aprendizaje y prácticas profesionales. La carga académica tiene un total de 216 créditos académicos, incluyendo 8 créditos de la práctica profesional. La secuencia de unidades de aprendizaje pretende una transmisión ordenada de la información al estudiante, la generación paulatina de capacidades analíticas y metodológicas, y la oportunidad para que el estudiante genere su propia síntesis de lo aprendido. En etapas avanzadas, los estudiantes pueden escoger materias optativas de Auditoria, Fiscal, Costos, Contabilidad y Finanzas. Además en forma extracurricular el alumno debe acreditar las competencias en Microsoft: Word, Excel y Power Point y cumplir con el Servicio Social de acuerdo con lo establecido en la Legislación Universitaria. Este es un programa educativo de calidad que ha sido evaluado en el nivel 1 por los Comités Interinstitucionales para la Evaluación de la Educación Superior, A. C. (CIEES) y también ha sido acreditado por el Consejo de Acreditación de la Enseñanza en la Contaduría y Administración, A. C. (CACECA) (Universidad Autónoma de Campeche, 2012).

\subsection{El Licenciado en Contaduría.}

El prototipo del Licenciado en Contaduría que se limitaba a cuadrar números, memorizar normas de contabilidad y escribir con lápiz en mano durante maratónicas jornadas de trabajo ha quedado atrás. En los tiempos actuales ha llegado a convertirse en un verdadero consultor, participe activo en las decisiones más importantes que definen el rumbo de las empresas, en pocas palabras ha llegado a ser un auténtico asesor de negocios (Ramos, 2009). La actividad principal del Licenciado en Contaduría se ha caracterizado por ser la de proveer un servicio profesional, sus servicios van enfocados a la generación de información relevante dirigida a diversas personas e instituciones coadyuvando con sus conocimientos y asesoría al desarrollo económico de las empresas.

Ramos (2010) señala que el Licenciado en Contaduría tiene que prepararse y desarrollarse en los nuevos ambientes cada vez más competitivos, en el cual conservará y desarrollará su propio campo de acción en la labor de fortalecer a las entidades en su administración, finanzas y 
control de las operaciones, apoyando con su capacidad profesional y responsabilidad social el crecimiento de las mismas para que puedan hacer frente a los nuevos retos que les depara el futuro. Montero y Zaa (2009) al referirse a la Contaduría Pública señalan que toda profesión no mercantil, basada en la práctica científica, evoluciona hacia formas más refinadas de prestación de servicios y que esto requiere un mayor control de calidad, no sólo para satisfacer la demanda de los clientes, sino para desarrollar de manera óptima, los métodos y procedimientos normativos por los que se rige el ejercicio profesional.

De acuerdo con Lopera, Granda, Castaño y Rodríguez (2009) el Licenciado en Contaduría tiene una importancia vital en el desarrollo de las Micro, Pequeñas y Medianas Empresas (Mipymes), tanto para la gestión como para todo lo relacionado con lo financiero. Desde la formación del Licenciado en Contaduría se hace énfasis en la importancia de representar la realidad con modelos y metodologías contables, matemáticas, financieras, fiscales, administrativas, sociales y económicas, todas estas como apoyo a la toma de decisiones para así brindar información de una manera óptima, clara y precisa.

El Licenciado en Contaduría desempeña diversas funciones empresariales como contador general de una empresa, consultor financiero y tributario. El Licenciado en Contaduría puede desempeñarse en el sector público y privado, por tanto su preparación es integral en todos los ámbitos empresariales. Antognolli (2008) manifiesta que la profesión del Licenciado en Contaduría los prepara fundamentalmente como asesores legales en materia comercial, laboral y especialmente impositiva, asimismo los forma para exponer informes ante organismos oficiales y realizar tareas de auditoría, entre otras. Por otra parte, en el contexto económico actual la necesidad de innovar, generar riqueza y desarrollar el potencial creativo se asocia con mayor fuerza a las Mipymes, las cuales deben estar provistas de una alta capacidad innovadora y una desarrollada capacidad emprendedora para sobrevivir y crecer. Sin embargo, poco se instruye a los Licenciados en Contaduría en el ámbito emprendedor.

\subsection{Planteamiento del problema.}


La capacidad emprendedora es una de las 15 competencias genéricas del perfil universitario para el nivel de licenciatura que la UAC ha definido. De las 13 competencias profesionales definidas para la Licenciatura en Contaduría, seis se refieren al área financiera, tres al área contable, dos al área de costos, una al área fiscal y la última sólo señala profundizar y facilitar los procesos de investigación, adopción e innovación tecnológica para incrementar la productividad y competitividad de las empresas. Debido a que no se tienen estudios sobre la manera en que están participando los nuevos Licenciados en Contaduría en el desarrollo de las Micro, Pequeñas y Medianas empresas (MiPymes), se decidió realizar el presente estudio de acuerdo con los siguientes objetivos.

\subsection{Objetivo general.}

Determinar si los Licenciados en Contaduría de la FCA de la UAC prefirieron crear sus propias fuentes de trabajo, o bien, trabajar por cuenta ajena en una empresa privada o en la administración pública.

\subsubsection{Objetivos específicos.}

a). Identificar los motivos de su decisión para trabajar en una empresa.

b). Identificar las fortalezas y debilidades de su preparación.

c). Determinar los principales problemas que enfrentan y cómo los manejan y resuelven.

1.5. Pregunta general de investigación.

¿Cuáles fueron las decisiones laborales y los principales motivos que tomaron los Licenciados en Contaduría de la FCA de la UAC en cuanto a crear sus propias fuentes de trabajo, o bien, trabajar por cuenta ajena en una empresa privada o en la administración pública?

1.5.1 Preguntas específicas de investigación. 
¿Cuáles son los motivos de su decisión para trabajar en una empresa?

¿Cuáles son las fortalezas y debilidades de su preparación?

¿Cuáles son los problemas que enfrentan y cómo los manejan y resuelven?

1.6 Hipótesis.

Los Licenciados en Contaduría de la FCA de la UAC no están creando empresas porque sus objetivos son básicamente de apoyo y asesoramiento en el desarrollo económico del estado de Campeche.

\subsection{Justificación.}

La investigación contribuye a la literatura acerca del comportamiento emprendedor y a encontrar las razones por las cuales los Licenciados en Contaduría de la FCA de la UAC prefieren dedicarse a una determinada actividad laboral, con la finalidad de proponer mejoras tendientes a fomentar la creación de nuevas empresas que contribuyan al desarrollo económico y sustentable del estado de Campeche y de México.

1.8 Limitaciones del estudio.

Este estudio sólo incluyó a los Licenciados en Contaduría que egresaron en el año de 2010

2. Método.

2.1. Tipo de estudio.

Este estudio fue exploratorio de corte cualitativo.

\subsection{Participantes.}


Participaron 70 Licenciados en Contaduría que egresaron en el año de 2010 de la FCA de la UAC. El $54 \%$ son mujeres y el $46 \%$ son hombres, con edades establecidas entre 22 y 31 años, el $13 \%$ se encontraba estudiando un posgrado.

\subsection{Instrumento.}

Se diseñó un cuestionario con preguntas semi estructuradas y se elaboró un análisis de la información documental. El instrumento inicial fue sometido a una prueba piloto permitiendo eliminar las preguntas poco claras o ambiguas y mejorar su planteamiento. El cuestionario finalmente quedó integrado en seis secciones: tres para empresarios y tres para trabajadores por cuenta ajena. Asimismo, se elaboró una guía de entrevistas con la finalidad de profundizar y encontrar explicaciones a los resultados obtenidos. El estudio cualitativo permitió "reforzar teorías e identificar cuestiones críticas para investigaciones futuras" (Stake, 1994, p. 245).

\subsection{Procedimiento.}

Para la aplicación de los cuestionarios, se solicitó el apoyo de la FCA de la UAC, obteniendo el listado de los Licenciados en Contaduría egresados en el año de 2010 y el apoyo de los alumnos en la recolección de datos. Los cuestionarios se administraron vía correo electrónico, además se realizaron entrevistas personalizadas y visitas a los diferentes centros de trabajo de los sujetos de estudio. La información se organizó mediante categorías y tablas de frecuencia permitiendo identificar y analizar los objetivos del estudio.

3. Resultados y discusión.

Con relación al objetivo general del estudio se encontró que 10 (14 \%) de los Licenciados en Contaduría trabajan de manera independiente o tienen una empresa propia y 60 (86 \%) trabajan por cuenta ajena. Las características generales de los sujetos del estudio se observan 
en la tabla 1.

Tabla 1

Datos generales de los Licenciados en Contaduría.

\begin{tabular}{lcccc}
\hline Categoría & Empresario & $\%$ & Cuenta ajena & $\%$ \\
\hline Sexo & 4 & 40 & 28 & 47 \\
Masculino & 6 & 60 & 32 & 53 \\
Femenino & & & 48 & 80 \\
Empresa & 4 & 40 & 7 & 12 \\
Servicios & 4 & 40 & 5 & 8 \\
Comercial & 2 & 20 & & \\
Industrial & & & 58
\end{tabular}

Nota: Elaboración propia.

En la tabla 1 se observa que 38 (54\%) de los encuestados son del sexo femenino, lo que significa que la profesión de Licenciado en Contaduría tiene una gran aceptación por parte de las mujeres. De los diez que se identificaron como empresarios se tiene la siguiente clasificación: a) tres prestan servicios de asesoría contable y financiera, b) uno se dedica a la renta de equipo para fiestas, c) cuatro están establecidos en el ramo comercial de compra venta de ropa, calzado y papelería, y d) dos se ubican en la venta de comida. De los 60 profesionistas que trabajan por cuenta ajena se tiene que: a) 48 (80\%) laboran en el sector servicios (22 en el gobierno, 12 en despachos, 6 en bancos y casas de préstamos, 4 en universidades, 2 en cámaras, 2 en hospitales); b) 7 (12 \%) trabajan en el sector comercial (3 a la venta de zapatos, ropa y papelería, 2 a la venta de material de construcción, 2 a la venta de aceites para motores); y c) 5 ( $8 \%$ ) al sector productivo ( 3 en maquiladoras y 2 en periódicos). Lo que indica que se tiene un nivel muy bajo de la actividad emprendedora y por tanto del desarrollo económico empresarial del estado de Campeche.

En cuanto al nivel de ingresos de los encuestados se especifican en la tabla 2. 
Tabla 2

Ingreso mensual de los Licenciados en Contaduría.

\begin{tabular}{lrrrr}
\hline Categoría & Empresario & $\%$ & Cuenta ajena & $\%$ \\
\hline$\$ 1,000-\$ 5,000$ & 5 & 50 & 29 & 48 \\
$\$ 6,000-\$ 10,000$ & 2 & 20 & 28 & 47 \\
$\$ 11,000-\$ 15,000$ & 1 & 10 & 3 & 5 \\
$\$ 16,000-\$ 20,000$ & 1 & 10 & & \\
$\$ 20,000$ - más & 1 & 10 &
\end{tabular}

Nota: Elaboración propia.

En la tabla 2 se aprecia que los ingresos mensuales de los empresarios fluctúan de $\$ 4,000.00$ a

$\$ 20,000.00$. Sólo uno manifestó ingresos superiores a los $\$ 20,000.00$. Los trabajadores por cuenta ajena comentaron que sus ingresos mensuales varían de $\$ 3,000.00$ a $\$ 15,000.00$. Se observa que 64 sujetos de estudio tienen ingresos hasta de $\$ 10,00.00$ y sólo seis tienen ingresos por arriba de esta cantidad.

Los puntos de vista de los entrevistados con relación a los motivos por los que muchos Licenciados en Contaduría prefieren buscar trabajo y no crear su propia empresa se detallan en la tabla 3. 
Tabla 3.

Motivos por los que muchos Licenciados en Contaduría prefieren buscar trabajo.

\begin{tabular}{lllllll}
\hline Categoría & Empresario & No. & $\%$ & Cuenta ajena & No. & $\%$ \\
\hline Preferencia & Recurso económico & 5 & 50 & Recurso económico & 40 & 67 \\
& Miedo & 1 & 10 & Miedo & 8 & 14 \\
& Negatividad & 1 & 10 & Experiencia & 6 & 10 \\
& Mentalidad & 1 & 10 & Mentalidad & 2 & 3 \\
& Desconocimiento & 1 & 10 & Oportunidad & 2 & 3 \\
& Comodidad & 1 & 10 & Iniciativa & 2 & 3 \\
\hline
\end{tabular}

Nota: Elaboración propia.

En la tabla 3 se nota que 5 (50\%) de los que se identificaron como empresarios señalaron como motivo principal por el que los Licenciados en Contaduría buscan trabajo es la falta de recursos económicos. Así como también, 40 (67 \%) de los que trabajan por cuenta ajena identificaron la misma causa por la que prefieren buscar trabajo. Existen otras causas que fueron mencionadas tales como el miedo, la negatividad, la falta de experiencia y la mentalidad entre otras.

En cuanto al primer objetivo específico, los Licenciados en Contaduría señalaron motivos concretos y personales que los llevaron a crear sus propias empresas, o bien, trabajar por cuenta ajena como se detallan en la tabla 4. 
Tabla 4.

Motivos de la decisión de los Licenciados en Contaduría para crear sus fuentes de trabajo o trabajar por cuenta ajena.

\begin{tabular}{lllllll}
\hline Categoría & Empresario & No. & $\%$ & Cuenta ajena & No. & $\%$ \\
\hline Motivos & Independencia & 4 & 40 & Experiencia & 19 & 32 \\
& Prosperar & 2 & 20 & Condiciones Trabajo & 15 & 25 \\
& Familia & 2 & 20 & Relación estudio & 14 & 24 \\
& Satisfacción & 1 & 10 & Oportunidad & 5 & 8 \\
& Falta empleo & 1 & 10 & Dificultad & 5 & 8 \\
& & & & Incertidumbre & 2 & 3
\end{tabular}

Nota: Elaboración propia.

En la tabla 4 se aprecia que a pesar de que en términos generales el recurso económico es el principal motivo que impide crear una empresa, 4 (40 \%) de los empresarios indicaron que siempre tuvieron la inquietud de independizarse y volverse autónomos ya que los obliga a ser más activos y a preocuparse por satisfacer a sus clientes. Otros motivos señalados fueron sus deseos de prosperar y el apoyo familiar. Por su parte, 19 (32 \%) de los encuestados que trabajan por cuenta ajena manifestaron que prefirieron emplearse en actividades de acuerdo a su perfil para aplicar sus conocimientos y adquirir experiencia. También indicaron que influyeron las condiciones de trabajo, la relación que tiene el trabajo que desempeñan con sus estudios y la oportunidad que se les presentó entre otros.

Con relación al segundo objetivo específico concerniente a las fortalezas y debilidades de su preparación, los Licenciados en Contaduría encuestados manifestaron los resultados que se indican en la tabla 5. 
Tabla 5.

Fortalezas y debilidades de los Licenciados en Contaduría.

\begin{tabular}{|c|c|c|c|c|c|c|}
\hline Categoría & Empresario & No. & $\%$ & Cuenta ajena & No. & $\%$ \\
\hline \multirow[t]{6}{*}{ Fortalezas } & Administración & 7 & 70 & Contabilidad & 24 & 40 \\
\hline & Proyectos & 2 & 20 & Fiscal & 13 & 22 \\
\hline & Finanzas & 1 & 10 & Proyectos & 8 & 14 \\
\hline & & & & Costos & 5 & 8 \\
\hline & & & & Taller emprendedor & 5 & 8 \\
\hline & & & & Finanzas & 5 & 8 \\
\hline \multirow[t]{5}{*}{ Debilidades } & Cómputo & 4 & 40 & Materias diversas & 27 & 45 \\
\hline & Actualización & 2 & 20 & Cómputo (Software) & 12 & 20 \\
\hline & Ninguno & 2 & 20 & Práctica & 10 & 17 \\
\hline & Incubadoras & 1 & 10 & Ninguno & 9 & 15 \\
\hline & Bursátil & 1 & 10 & Actualización & 2 & 3 \\
\hline \multirow[t]{5}{*}{ Componentes } & T. Emprendedor & 4 & 40 & T. emprendedor & 19 & 32 \\
\hline & Software & 3 & 30 & Práctica Cont. y Fin & 19 & 32 \\
\hline & Finanzas & 1 & 10 & Convenios & 12 & 20 \\
\hline & Proyectos & 1 & 10 & Software & 7 & 11 \\
\hline & Actualización & 1 & 10 & Proyectos & 3 & 5 \\
\hline
\end{tabular}

Nota: Elaboración propia.

En la tabla 5 se percibe que 7 (70 \%) de los egresados que tienen sus propias empresas indicaron como fortalezas las materias de Administración y 2 (20 \%) la elaboración de proyectos. Es importante notar que 24 (40 \%) de los que trabajan por cuenta ajena identificaron las materias de Contabilidad y 13 (22 \%) los aspectos fiscales como fortalezas, debido a que son las que más utilizan en la organización donde trabajan. 
Por otra parte, 4 (40 \%) de los empresarios manifestaron como debilidades la falta de conocimientos de cómputo y $2(20 \%)$ la falta de actualización; mientras que 27 (45\%) de los egresados que trabajan por cuenta ajena señalaron como debilidades diversas deficiencias en materias que cursaron y $12(20 \%)$ indicaron la falta de conocimientos de software de cómputo.

Asimismo, 4 (40 \%) de los empresarios coincidieron con 19 (32\%) de los trabajadores por cuenta ajena, en incluir la asignatura de Taller de Emprendedores en los programas de estudio de licenciatura para que los egresados puedan crear o iniciar sus propias empresas. Otros componentes que consideraron importantes incluir fueron los Software Contables y de Cómputo. Cabe hacer notar que 19 (32\%) consideraron indispensable tener en cuenta las asignaturas de Prácticas Contables y Financieras 19 (32) y la celebración de Convenios institucionales 12 (20\%), entre otros.

Con respecto al tercer objetivo del estudio, relativo a los problemas para iniciar una empresa y como los resuelven o manejan, los resultados se señalan en la tabla 6 . 
Tabla 6.

Problemas y como los resuelven los Licenciados en Contaduría.

\begin{tabular}{lllllll}
\hline Categoría & Empresario & No. & $\%$ & Cuenta ajena & No. & $\%$ \\
\hline Problemas & Recurso económico & 4 & 40 & Recurso económico & 36 & 60 \\
& Fiscales & 3 & 30 & Experiencia & 7 & 12 \\
& Burocracia & 2 & 20 & No ha intentado & 7 & 12 \\
& Apoyo gobierno & 1 & 10 & Burocracia & 5 & 8 \\
& & & & Inestabilidad & 5 & 8 \\
& & & & & & \\
Resuelven & Ahorro & 2 & 20 & No ha intentado & 21 & 35 \\
& Apoyo familiar & 2 & 20 & Investigando & 12 & 20 \\
& Administrando & 2 & 20 & Ahorro & 14 & 23 \\
& Tiempo & 2 & 20 & Trabajando & 10 & 17
\end{tabular}

Nota: Elaboración propia.

En la tabla 6 se advierte que 4 (40\%) de los empresarios manifestaron como principal problema el recurso económico, también fueron señalados problemas fiscales, burocráticos y la falta de apoyo del gobierno. También se observa que 36 (60\%) de los trabajadores por cuenta ajena coincidieron con los empresarios al afirmar que su principal problema es el recurso económico.

Asimismo, los empresarios resuelven sus problemas de diferentes maneras tales como: ahorro, apoyo familiar, administrando, tiempo y crédito; mientras que 21 (35\%) de los trabajadores por cuenta ajena no han intentado iniciar una empresa y 39 (65 \%) se encuentra investigando, ahorrando, trabajado o adquiriendo experiencia. 
Finalmente, se tomaron en consideración las recomendaciones de los encuestados con base en sus experiencias, obteniendo los resultados de la tabla 7.

Tabla 7.

Recomendaciones de los Licenciados en Contaduría.

\begin{tabular}{lllllll}
\hline Categoría & Empresario & & No. & Cuenta ajena & No. & $\%$ \\
\hline Recomendaciones & Emprendedores & 5 & 50 & Emprendedores & 33 & 55 \\
& Convenios & 3 & 30 & Incubadoras & 18 & 30 \\
& Actualización & 2 & 20 & Práctica profesional & 7 & 12 \\
& & & & Actualización & 2 & 3
\end{tabular}

Nota: Elaboración propia.

En la tabla 7, se distingue que las recomendaciones de los empresarios consisten en fomentar la cultura emprendedora, los convenios y la actualización, situación que coincide con los trabajadores por cuenta ajena, pues estos recomendaron fomentar la cultura emprendedora, creación de incubadoras, práctica profesional y actualización.

\section{Conclusiones}

Los principales factores que influyen para que un Licenciado en Contaduría inicie su propia empresa son sus deseos de independencia y prosperidad, el apoyo familiar y en ocasiones la falta de empleo. Mientras que los factores que influyen para que trabaje por cuenta ajena son: la falta de experiencia, la oferta de trabajo durante sus estudios y la comodidad que ofrecen las condiciones de trabajo tales como sueldo fijo, prestaciones y prima de antigüedad entre otras. Los Licenciados en Contaduría consideran que las materias del área de Administración, Contabilidad, Fiscal y Proyectos constituyen una fortaleza, pero que también existen algunas deficiencias por la forma en que fueron impartidas algunas materias o bien, les faltó actualización, lo que consideraron como una debilidad. En cuanto a los componentes necesarios 
para coadyuvar la creación de sus propias empresas es importante vincular más a fondo el ambiente emprendedor, el uso de software contables y mayor práctica contable y financiera.

Para resolver sus problemas de financiamiento, los empresarios están ahorrando, o bien, reciben apoyo familiar, lo que es consistente con la literatura al afirmar que uno de los principales problemas en América Latina es la falta de financiamiento barato (América Economía, 2003). Asimismo, mediante el crédito, el tiempo y una buena administración resuelven diversos problemas. Los trabajadores por cuenta ajena están investigando, ahorrando, trabajando y adquiriendo experiencia para resolver sus problemas mediante la espera de una oportunidad y señalaron que la falta de capital, la falta de experiencia, la burocracia y la inestabilidad económica son los principales problemas para emprender, lo que no es consistente con la literatura de investigación ya que para hacer un negocio más que riquezas se requiere de gran empeño. Cabe mencionar que también hay quienes nunca han tenido el interés de crear una empresa, no es su prioridad y están contentos con su situación de trabajadores por cuenta ajena. En este sentido opinaron que no todos los profesionistas tienen que ser emprendedores para ser socialmente responsables y contribuir al desarrollo económico del estado de Campeche y de México.

Asimismo, es recomendable fomentar la cultura emprendedora en diferentes direcciones: a) vinculación con el sector empresarial, b) incubadora de negocios, c) convenios empresariales, d) práctica profesional, y e) participación en congresos y eventos, entre otros. Asimismo, es indispensable realizar una revisión y actualización de los programas de estudio, pues mucho de lo que se enseña ya está obsoleto y no tiene aplicación real.

Debido a que este estudio es de carácter exploratorio, mayor investigación es necesaria de tal manera que puedan obtenerse resultados más sólidos para lograr establecer mayores diferencias entre los Licenciados en Contaduría que son empresarios y los que trabajan por cuenta ajena. Otros estudios podrían identificar si los requerimientos actuales de las empresas 
coinciden con los conocimientos que se imparten a los profesionistas, en beneficio del desarrollo económico del estado de Campeche y de México.

\section{Bibliografía}

América Economía (2003). El error de los emprendedores es querer hacerlo todo. Clima de emprendimiento organizado (ceo.cl). Entrevista con J. Schulman. Recuperado de http://www.ceo.cl/609/article-33507.html

Antognolli, S. (2008). El Contador Público en las PYME. Gestiopolis.com. Recuperado de http://www.gestiopolis.com/innovacion-emprendimiento/contador-publico-en-laspymes.htm

Montero, H. y Zaá, R. (2009). Ética, normatividad y calidad. XXVIII Conferencia Interamericana de Contabilidad. [Documento en PDF]. Recuperado de http://www.colegiodecontadorescochabamba.org.bo/descargas/octubre09total.pdf

Stake, Robert. (1994). Case studies. Thousand Oaks: CA. 